\title{
Tendencias didácticas de algunos docentes universitarios de matemáticas
}

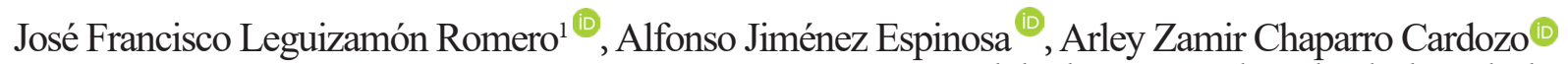
Universidad Pedagógica y Tecnológica de Colombia- Colombia

Autor de correspondencia: ${ }^{1}$ francisco.leguizamon@uptc.edu.co

Recibido: 01 de agosto de 2019, Revisado: 30 de octubre de 2019 Aprobado: 28 de febrero de 2020 Publicado: 24 de mayo de 2020

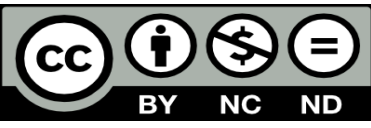

\section{Resumen}

Se presenta un análisis sobre el quehacer docente de profesores de la Licenciatura en Matemáticas de la Universidad Pedagógica y Tecnológica de Colombia, que puede considerarse como un punto de partida para mejorar las prácticas de aula. El enfoque teórico es el de modelos de clase propuesto por Porlán, complementado con el constructivismo clásico de Piaget, el enfoque sociocultural de Vygotsky y el interaccionismo de Bruner. La metodología de investigación es mixta, con alcance descriptivo. Los instrumentos de recolección de información fueron los cuestionarios y la entrevista no estructurada. Los resultados muestran que la mayoría de los docentes que participaron en el estudio: diseñan sus clases con base en objetivos operacionales; evalúan mediante la aplicación de pruebas objetivas, planteadas especialmente como guías; y consideran que el aprendizaje ocurre mediante un proceso progresivo de asimilación de concepto. De acuerdo con lo anterior, se evidencia que las características de las prácticas de los profesores se asocian con una tendencia didáctica tecnológica.

Palabras clave: enseñanza de las matemáticas, profesor universitario, tendencias didácticas 


\title{
Didactic tendencies of some university mathematics teachers
}

\begin{abstract}
The article presents an analysis of the teaching work of teachers of the Degree in Mathematics of the Pedagogical and Technological University of Colombia, which can be considered as a starting point to improve classroom practices. The theoretical approach is that of class models proposed by Porlán, complemented by Piaget's classical constructivism, Vygotsky's socio-cultural approach, and Bruner's interactionism. The research methodology is mixed, with a descriptive scope. The instruments used to collect information were questionnaires and unstructured interviews. The results show that most of the teachers who participated in the research: design their classes based on operational objectives; assess through the application of objective tests, posed especially as guides; and consider that learning occurs through a progressive process of concept assimilation. According to the aforementioned, it is evident that the characteristics of teachers' practices are associated with a technological didactic tendency.
\end{abstract}

Keywords: mathematics education, university teacher, teaching tendencies

\section{Tendências didáticas de alguns docentes universitários de matemática}

\section{Resumo}

$\mathrm{O}$ artigo apresenta uma análise sobre o afazer docente de professores do Bacharelado em Matemática da Universidade Pedagógica e Tecnológica da Colômbia, que pode ser considerada como ponto de partida para melhorar as práticas na sala de aula. A abordagem teórica é a dos modelos proposta por Porlán, complementada pelo construtivismo clássico de Piaget, a abordagem sociocultural de Vygotsky e o interacionismo de Bruner. A metodologia de pesquisa é misturada, com alcance descritivo. Os instrumentos de colheita da informação foram os questionários e a entrevista não estruturada. Os resultados mostram que a maioria dos docentes que participaram do estudo: planejam suas aulas com base em objetivos operacionais; avaliam através da aplicação de testes objetivos, planteados especialmente como guias; e consideram que a aprendizagem ocorre através de um processo progressivo de assimilação de conceito. De acordo com o acima exposto, evidencia-se que as características das práticas dos professores estão associadas a uma tendência didática tecnológica.

Palavras-chave: ensino de matemática, professor universitário, tendências didáticas 


\section{Introducción}

Este artículo se basa en los resultados parciales de la investigación de Leguizamón (2017a), en el que se analizan las prácticas docentes de un grupo de profesores de la Licenciatura en Matemáticas de la Universidad Pedagógica y Tecnológica de Colombia [UPTC]. Este puede considerarse como un diagnóstico inicial para proponer estrategias de mejoramiento de las prácticas de aula, pues es de amplio conocimiento que las matemáticas siguen siendo consideradas por los estudiantes como una de las materias más difíciles de aprender en los currículos escolares. Una de las causas de dicha dificultad radica en las acciones de los docentes y sus concepciones sobre la asignatura. Por esto, la investigación en su primera fase, identifica tendencias didácticas predominantes de algunos docentes de la licenciatura, de manera que después puedan ser contrastadas con situaciones de aula y contribuyan a que el docente reconozca las características de su práctica y pueda establecer acciones para mejorarla.

En la literatura académica hay muchas clasificaciones de modelos pedagógicos y didácticos que dependen de los intereses y del objeto de investigación de cada autor. Por ello, se escogieron algunos que, aunque son genéricos para cualquier área, se pueden enfocar hacia la matemática y la clase de matemáticas (Leguizamón, 2017b). Inicialmente, se considera la propuesta de Ernest (1989), quien afirma que las concepciones acerca de la naturaleza de las matemáticas, la enseñanza y su proceso de aprendizaje son factores determinantes en las prácticas del docente. Además, se analiza la propuesta de Porlán (1995), junto con el constructivismo clásico de Piaget, el enfoque sociocultural de Vygotsky y el interaccionismo de Bruner. En consecuencia, para explicar las acciones del docente, se usó el término tendencia, pues un docente no se ubica en un solo modelo, ya que se evidencian características de muchos. Sin embargo, una mayoría de características - de uno o varios de los modelos- subyace en sus prácticas.

El estudio es exploratorio. El propósito es problematizar la práctica docente cotidiana, para conocer y caracterizar el principal modelo didáctico asumido por docentes de la licenciatura. Para lograrlo, la metodología de la investigación se diseñó desde un enfoque mixto, con alcance descriptivo. Como instrumentos de recolección de información se usaron dos cuestionarios y una entrevista no estructurada.

\section{Algunas tendencias didácticas en educación matemática}

Debido a la variada clasificación de modelos pedagógicos en la literatura académica, se buscaron los que permitieran comprender de manera clara las prácticas pedagógicas de docentes de matemáticas.

\section{Enfoque tradicional.}

Se presenta cuando el docente considera que solo hay una forma de desarrollar el trabajo en el aula. Enseñar se asume como explicar contenidos básicos a estudiantes, mientras se definen significados y, en algunas oportunidades, se presentan ejemplos o demostraciones. El docente se preocupa por desarrollar contenidos organizados secuencialmente y asume que ese es el conocimiento que el estudiante necesita de la disciplina en un determinado grado. En la mayor parte de la clase, el profesor explica y el alumno escribe la información (Jiménez, 2019a). 
Una gran cantidad de docentes sigue este modelo, pues afirman que "es la única forma" en que saben desarrollar su trabajo (Porlán, 1995, p. 146). Este modelo se manifiesta en las prácticas docentes por diversas razones, como los sistemas de organización y gestión académica de las instituciones educativas estancadas en los paradigmas fordistas y lo que la sociedad espera de las prácticas docentes. Sin embargo, la causa más frecuente es que los docentes repiten los modelos de clase de cuando eran estudiantes (Jiménez, Limas \& Alarcón, 2016).

La transmisión verbal de conocimientos es una práctica generalizada, lo que no implica que sea la mejor, pues en la mayoría de los casos no consigue un adecuado aprendizaje. De acuerdo con Porlán (1995), algunos aspectos negativos de esta tendencia didáctica son: se pierde la motivación de los estudiantes al establecerse las temáticas de manera impositiva por parte del docente; se vende la idea de una matemática terminada, pues se plantean los contenidos como unidades de verdad; el profesor siempre culpa al estudiante por el fracaso de su aprendizaje, ya que la premisa es que, si el profesor explica adecuadamente, el estudiante debe aprender (Jiménez, 2019a); los estudiantes tienden a preparar mecánicamente las evaluaciones, ya que casi siempre en ellas se busca que memoricen.

\section{Enfoque tecnológico.}

Su pretensión es superar el reduccionismo en los procesos didáctico y metodológico presentados por el enfoque tradicional. Se destaca porque toda práctica educativa se realiza con una intencionalidad, o sea el logro de unos objetivos. En cuanto al aprendizaje, Porlán (1995) plantea que sucede por un proceso de asimilación de conceptos con niveles crecientes de dificultad. Pretende hacer una evaluación objetiva del progreso de los aprendizajes del estudiante con el fin de determinar la recuperación de los aspectos no exitosos detectados en la prueba diagnóstica inicial. También se realiza una prueba diagnóstica final. Las pruebas objetivas se realizan con test de opción múltiple.

Al igual que el enfoque tradicional, presenta algunas fallas: la idea de eficacia se convierte en una obsesión eficientista, rígida y uniformizadora (Gimeno, 1982, citado por Porlán, 1995, p. 153); los objetivos se tornan rígidos si no se comprende que deben ser replanteados según las necesidades e intereses de los estudiantes; la evaluación, al ser cerrada, favorece aprendizajes mecánicos; y genera problemas de rigidez en las prácticas, causados por las secuencias de actividades cerradas y la falta de motivación.

\section{Tendencia espontaneísta -o activista-.}

Busca posicionar al estudiante como el centro del proceso para que pueda tomar decisiones sobre qué y cómo aprender en un ambiente agradable y natural, lo que le permite realmente interesarse, al ser también un ente organizador. La labor del docente es de orientador, coordinador de actividades de iniciativa del estudiante, mientras apoya la interacción y comunicación entre todos los alumnos; pero, ante todo, es un improvisador permanente, sin tomar en ningún momento conductas calificadoras o sancionadoras. Como el estudiante es el eje del proceso de enseñanza, se asume que este modelo está contrapuesto a los anteriores, donde el eje es el docente, lo cual pone de manifiesto uno de los problemas históricos de la educación: los estudiantes mentalmente separan los significados académicos de los significados de la vida cotidiana (Porlán, 1995). 
Algunas de las características propias del enfoque espontaneísta son: no existe una programación definida, ni contenidos claros y específicos, ni completa negociación con los estudiantes de proyectos a trabajar, los cuales pueden ser orientados a todo el grupo o a pequeños grupos; se da especial importancia a las salidas de observación, actividades de consenso y comunicación; el plan de trabajo se adecúa según los intereses de los estudiantes; y periódicamente se realizan asambleas con el fin de discutir sobre la dinámica del aula (Porlán, 1995).

\section{Constructivismo.}

El principal representante del constructivismo clásico es Jean Piaget, según el cual para que un aprendizaje sea efectivo debe ser activo (Sierpinska, 1998), es decir, la persona aprende mediante su acción sobre los objetos de conocimiento. Las interacciones con el contexto no son suficientes para obtener el desarrollo cognitivo, ya que este depende de la maduración biológica de las funciones cerebrales, condición para el aprendizaje. La escuela contribuye al desarrollo, pero por sí sola no lo puede lograr (Chaparro \& Leguizamón, 2015).

Piaget (1978) plantea cuatro estadios de aprendizaje del ser humano: sensorio-motor, pre-operatorio, de las operaciones concretas y de las operaciones formales; los cuales describen los procesos de desarrollo de las potencialidades del individuo (Sierpinska, 1998).

Desde el constructivismo, el alumno construye su propio conocimiento. El discurso del profesor se asume como enseñanza directa que impide al alumno actuar sobre los objetos de conocimiento, por lo cual el alumno no puede aprender a través de este (Chaparro \& Pachón, 2016). Por lo anterior, Sierpinska (1998) concluye que bajo la perspectiva constructivista no es viable compartir el conocimiento a través de la comunicación oral entre alumno y profesor, ni entre alumno y compañeros. Desde esta perspectiva teórica, el proceso comunicativo es muy limitado.

\section{Tendencia sociocultural.}

Lev Vygotsky es considerado como su principal representante, el cual fue influenciado por la concepción marxista. Él otorga gran importancia a las prácticas sociales en el aprendizaje. En oposición a Piaget, Vygotsky considera independientes a la educación y al desarrollo, pues este último lo plantea como un proceso de enculturación.

Según Sierpinska (1998), Vygotsky destaca la mediación simbólica como base para la comprensión del desarrollo humano, como proceso sociocultural. De acuerdo con lo anterior, el hombre no tiene acceso directo a los objetos, pero sí a través de los sistemas simbólicos de que dispone. Por esto, el enfoque plantea la construcción del conocimiento como una interacción mediada por relaciones y por otros sujetos, como la organización del ambiente y el mundo cultural, según el contexto de la persona.

Para Vygotsky (1995), la interacción entre pares o con un compañero más experimentado es determinante para obtener aprendizajes significativos. Este autor define la zona de desarrollo próximo como la distancia entre el desarrollo real de una persona y su desarrollo potencial. La interacción entre pares en esa zona facilita la comunicación y, por ende, el aprendizaje (Jiménez, 2019b). 
Vygotsky, al darle importancia al desarrollo del pensamiento, se preocupa también por el estudio del lenguaje escrito, ya que, según Sierpinska (1998), el factor más importante del lenguaje escrito es su carácter voluntario, es decir, es realizado conscientemente mediante un sistema de signos escogidos voluntariamente por el individuo. Vygotsky prioriza el uso de los símbolos siempre que estén ligados a las prácticas culturales. Afirma que pueden convertirse en una herramienta importante para el aprendizaje.

\section{Tendencia interaccionista.}

Uno de sus principales representantes es Jerome Bruner, quien resalta el papel de las interacciones sociales en el aprendizaje. Él desafió el behaviorismo, el cual aún tiene fuertes manifestaciones en las clases de matemáticas. En esta tendencia, el individuo que aprende selecciona y transforma la información, construye hipótesis y toma decisiones de acuerdo con su estructura cognitiva, la cual le permite asignar significado y organización a las experiencias, lo que implica que el aprendiz vaya más allá de la información recibida. Bruner (1990) afirma que el aprendizaje es un proceso que ocurre internamente y mediado cognitivamente; no es un producto directo del ambiente o de factores externos al aprendiz. Sí tiene en cuenta los aspectos sociales y culturales, pero les da menos importancia que Vygotsky.

Aunque las teorías de Piaget y de Bruner basan sus investigaciones en el desarrollo infantil, Bruner prioriza el lenguaje, la cultura y las técnicas como medios que viabilizan le emergencia de formas de representación. Afirma que el desarrollo cognitivo será más rápido en cuanto se tengan más y mejores formas de acceso a un medio cultural estimulante (Marques, 2013). La teoría de Bruner asume el maduracionismo de Piaget y el ambientalismo de Vygotsky, pues considera que es a partir de allí como el individuo organiza los modos de representación, pero mediados por la cultura.

Bruner clasifica el desarrollo cognitivo en tres estadios: el de las respuestas motoras, el de la representación icónica y el de las representaciones simbólicas. El paso de estas etapas puede hacerse más ágil si el niño se desarrolla en un contexto cultural rico (Marques, 2013).

Desde esta teoría, cuando el profesor de matemáticas aborda un concepto, no está comunicándolo a los alumnos, al igual que los alumnos no están comunicando sus conceptos construidos social, histórica y culturalmente, ya que los significados no están en la mente de las personas para ser transmitidos de una persona a otra. Cuando dialogan el profesor y los estudiantes en la clase, ocurre una interacción y el conocimiento matemático emerge de esa interacción (Sierpinska, 1998).

Principios de pedagogía conceptual.

Esta tendencia, creada por Zubiría (2007), busca un cambio de una concepción epistemológica empirista a una racionalista. Se pretende desarrollar las áreas de talento de cada estudiante, al rescatar que lo más importante de él no es su capacidad de memoria, sino sus procesos cognitivos y afectivos. Igualmente, lo más destacable del maestro es su capacidad para desarrollar la mente humana, pues su compromiso es enseñar y ser un modelo de ser humano, con autoridad. Esta tendencia se basa en tres sistemas. En los sistemas cognitivos se busca que el estudiante aprehenda los instrumentos de conocimiento -noción, proposición y pensamiento-, mientras que las operaciones intelectuales 
que se privilegian son analizar, inducir, deducir... Los sistemas afectivos son aquellos donde se utilizan instrumentos afectivos como emociones, sentimientos, actitudes, valores... Finalmente, mediante los sistemas expresivos se convierten los pensamientos y sentimientos a lenguaje. En conclusión, la pedagogía conceptual busca formar un ser humano equilibrado afectivamente, destacado cognitivamente y talentoso a la hora de expresarse (Zubiría, 2006).

\section{Metodología}

Asumir un determinado paradigma de investigación no es gratuito, ni a la ligera, ya que el problema de investigación y el paradigma son interdependientes (Strauss \& Corbin, 1990). Lo primero que debe buscar el investigador es una armonización entre ellos de acuerdo con su visión investigadora. Dado que los fenómenos y problemas que enfrentan las ciencias sociales y humanas son cada vez más complejos y diversos, se ha visto la necesidad de utilizar un enfoque mixto de investigación (Morse, 2003, citado por Tashakkori \& Teddlie, 2003), el cual logra una perspectiva más amplia y profunda de la situación a estudiar, al facilitar una mejor exploración y explotación de los datos (Hernández, Fernández \& Baptista, 2014). Se asumió este enfoque de investigación, dado que se requirió manejar datos tanto cualitativos como cuantitativos y existió la necesidad de realizar un análisis que incluyera las dos visiones.

En la investigación descriptiva, el objetivo fundamental es recopilar datos e información sobre las características, propiedades, aspectos, clasificaciones, personas, agentes e instituciones de los procesos naturales o sociales, para conocer cómo se constituye un fenómeno; es decir, "un estudio descriptivo determina e informa los modos de ser de los objetos" (Gay, 1996 citado por Naupas, Mejía, Novoa \& Villagómez, 2014, p. 92). Este tipo de investigación es utilizada como diagnóstico, donde se responde a preguntas tales como: cuál es la relación entre, cómo es, qué diferencias existen, cómo se comporta, cómo se clasifica; es decir, se utiliza como paso inicial para cualquier investigación de mayor envergadura.

El estudio completo se desarrolló en tres momentos: exploración, intervención y evaluación. Acá se presenta el análisis de la etapa de exploración, que tiene como propósito problematizar la práctica docente cotidiana, para conocer y caracterizar el principal modelo didáctico asumido por docentes del programa de la licenciatura. La metodología de la investigación se diseñó desde un enfoque mixto, con alcance descriptivo.

La licenciatura cuenta con nueve profesores de tiempo completo adscritos directamente y quince profesores de tiempo completo vinculados a la Escuela de Matemáticas y Estadística de la Facultad de Ciencias, al igual que con veinte catedráticos. El estudio se realizó con profesores de tiempo completo que desarrollan su actividad docente con los estudiantes de la licenciatura, quienes conformaron la unidad de análisis.

Para la recolección de información, se usaron tres instrumentos. El cuestionario uno consta de 30 preguntas cerradas, elaboradas a partir de la clasificación de Porlán (1995). El objetivo de su aplicación fue identificar las tendencias pedagógicas de los docentes. Este instrumento fue validado y aplicado por Díaz (2010) y analizado por los investigadores. El cuestionario dos tiene 38 preguntas cerradas, las cuales fueron adaptadas de Martínez (2003), quien originalmente 
lo validó. Sin embargo, antes de su aplicación, se realizaron pruebas piloto para identificar las concepciones de los docentes de la licenciatura acerca de: los objetivos de enseñanza, significado de la temática, importancia que le da el docente al contexto, cómo aborda las dificultades en el aprendizaje, las interacciones en el aula, formas de representación, medios educativos, relación entre la comprensión y la mecanización, entre otros (Leguizamón, Patiño \& Suárez, 2015). En tercer lugar, la entrevista no estructurada se aplicó como complemento a los dos cuestionarios anteriores. Su objetivo fue ampliar la información suministrada por los profesores en los instrumentos, por ello no se separa su análisis, sino que se realiza transversalmente con cada cuestionario.

\section{Resultados}

\section{Cuestionario uno.}

Estuvo orientado a los docentes que trabajan en la licenciatura. Este cuestionario fue aplicado por Díaz (2010) a 23 docentes, tanto de la Escuela de Matemáticas y Estadística como de la licenciatura, con el requisito que todos trabajaran cursos con los estudiantes de la licenciatura. Se plantearon seis preguntas de cada tendencia: tradicional, tecnológica, espontaneísta, principios de pedagogía conceptual y constructivista. Se realizó un análisis, pregunta a pregunta, para saber cuántos profesores habían seleccionado la opción A - si está de acuerdo con la tendencia y habitualmente la aplica-. El resultado obtenido se plasma en la tabla 1. Los docentes fueron codificados con los números del 1 al 23.

Tabla 1

Los docentes y su clasificación de acuerdo con Díaz (2010)

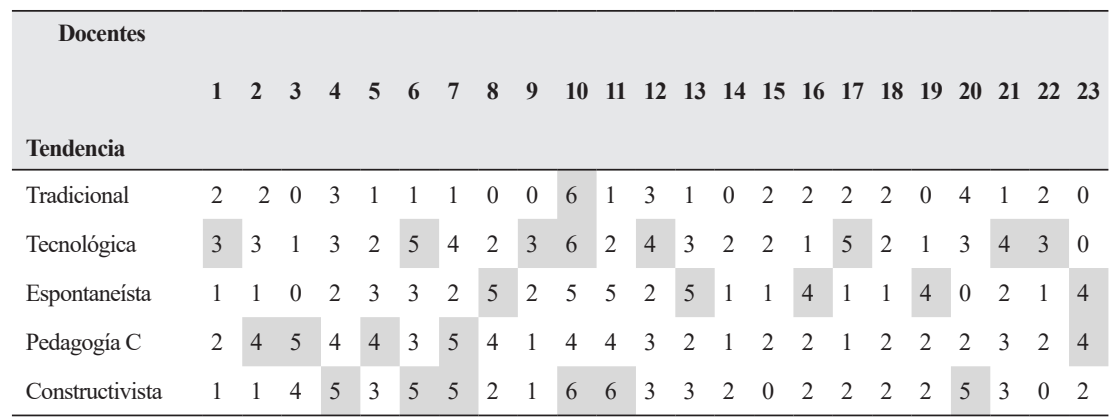

Fuente: elaboración propia.

De la anterior información, se puede concluir que ninguno de los 23 profesores asume su práctica docente desde la tendencia didáctica tradicional. Los profesores P1, P9, P12, P17, P21 y P22 presentan tendencia didáctica tecnológica. Se acercan a la tendencia espontaneísta los docentes P8, P13, P16 y P19. En la tendencia hacia la pedagogía conceptual están los docentes P2, P3 y P5. Finalmente, los docentes que presentan tendencia constructivista son P4, P11 y P20. No se puede definir la tendencia didáctica de los profesores P6, P7, P10, P14, P15, P18 y P23. En resumen, en cuanto a las tendencias didácticas a las cuales se aproximan los docentes, se evidenció que el $26 \%$ presenta tendencia tecnológica, el $17 \%$ tendencia espontaneísta, el $13 \%$ tendencia hacia la pedagogía conceptual, el 13\% tendencia constructivista. No se pudo definir la tendencia del $31 \%$, ya que presenta igualdad de condiciones para varias tendencias o no predominan las características de una tendencia en particular. Pese a que una cantidad 
significativa del grupo de profesores asume su práctica desde una tendencia tecnológica, ningún docente mostró pertenecer a una tendencia exclusiva, es decir, no reconoció en su práctica todas las características específicas que componen una determinada tendencia, de las planteadas por Porlán (1995).

De los datos se infiere que prima la tendencia tecnológica, cuyas características, según la encuesta y la entrevista, son: a) la necesidad de planificar rigurosamente la clase con especial cuidado al proponer los objetivos, considerando elementos importantes en el currículo, los cuales deben ser planteados jerárquicamente, al esbozar primero los conocimientos más concretos y para progresivamente ir hacia lo más complejo; b) una forma de ejercer control sobre el aprendizaje es analizar las conductas observables determinadas por los objetivos; c) un estudiante no realiza correctamente las tareas de la clase porque no tiene interés o voluntad para trabajar, o su inteligencia está por debajo de lo normal, es decir, hay una concepción de aprendizaje homogenizado: el profesor explica y todos aprenden de la misma manera.

\section{Cuestionario dos.}

Estuvo orientado a profesores, con el fin de determinar metodologías de enseñanza y de aprendizaje. Inicialmente, las 38 preguntas fueron distribuidas en las siguientes categorías: objetivos -4 preguntas -, significado de la temática $-7-$, importancia del contexto $-7-$, dificultades en el aprendizaje -2-, importancia de los conocimientos previos - 3-, interrelación entre estudiantes - 2-, representación -2-, medios educativos -2-, operación y su inversa $-2-$, ejercicio y problema $-2-$, comprensión-mecanización $-2-$ y relación conocimiento personal-conocimiento institucional -3-. Los resultados más destacados se plantean en la tabla 2 .

Tabla 2

Los docentes y las respuestas más usuales de acuerdo con las categorías ${ }^{1}$

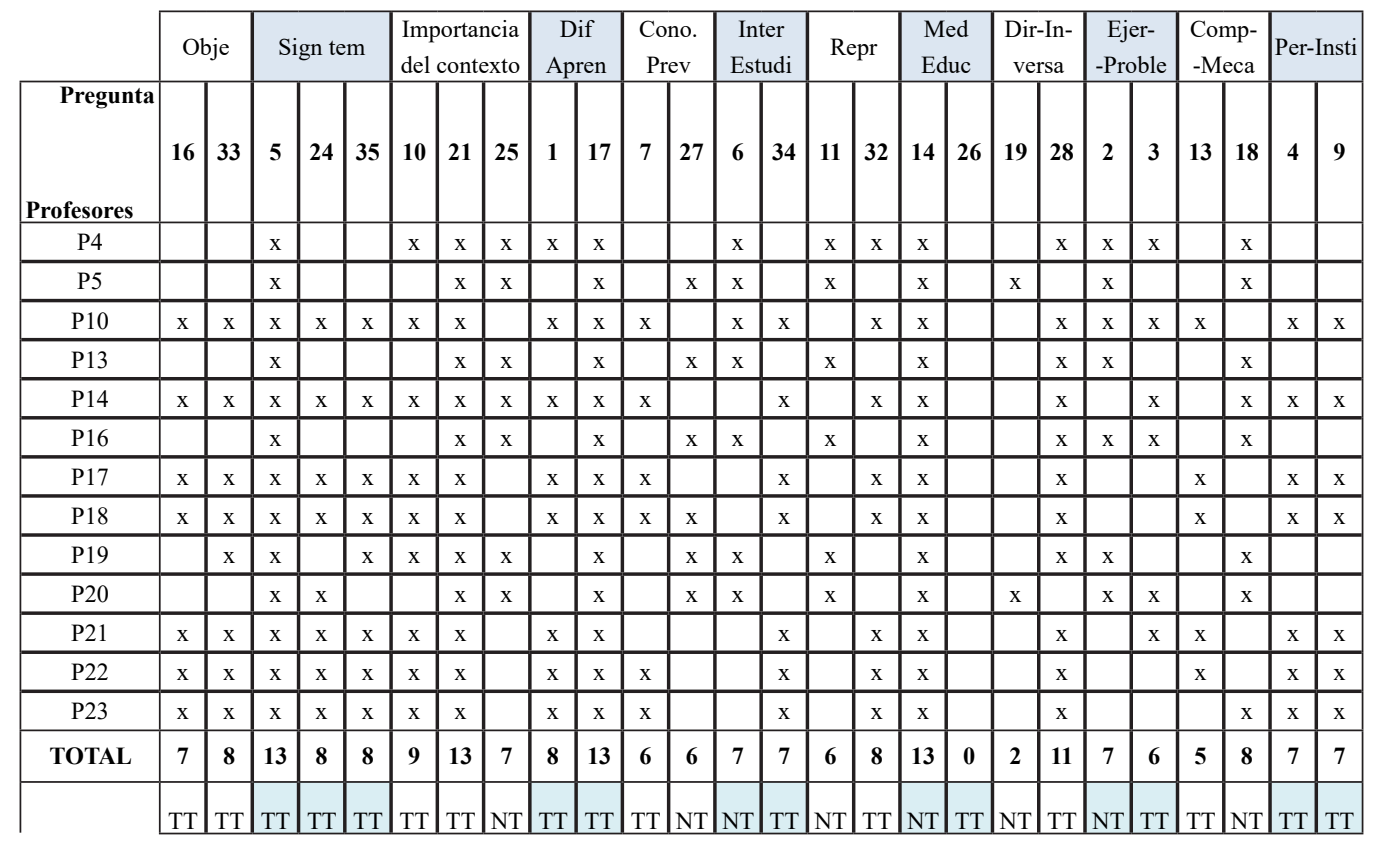

Fuente: elaboración propia.

1. Nota. NT: tendencia no tradicional-tecnológica; TT: tradicional-tecnológica. 
$\mathrm{Al}$ analizar las categorías teóricas, se encontró que algunas de las tendencias tenían características en común, lo cual dificultaba su clasificación. Por tal razón, los investigadores decidieron reagrupar las tendencias y plantear una nueva categorización. Como nuevas categorías se tomaron las tendencias centradas en el profesor - tradicional-tecnológica [TT] y las tendencias centradas en el estudiante - no tradicional-tecnológica [NT] -, es decir, espontaneísta, pedagogía conceptual y constructivista. Los resultados de la tabla 2 son los siguientes:

\section{Objetivos.}

- Afirmación: "La enseñanza de una temática se hace para solucionar problemas de la vida cotidiana".

Docentes de acuerdo: P14, P17, P18, P19, P21, P22 y P23 — 54\%—.

Tendencia: TT.

- Afirmación: "Es importante desarrollar habilidad para solucionar ejercicios".

Docentes de acuerdo: P14, P17, P18, P19, P21, P22 y P23 — 54\%-.

Tendencia: TT.

\section{Significado de la temática.}

- Afirmación: "Para que un estudiante comprenda el significado de una temática se deben proponer problemas que se solucionen con ella".

Docentes de acuerdo: todos.

Tendencia: TT.

- Afirmación: "Para que un estudiante solucione un problema, tiene que desarrollar ejercicios primero, los cuales deben ser propuestos en forma graduada por el docente".

Docentes de acuerdo: P10, P14, P17, P18, P21, P22 y P23 — 54\%—.

Tendencia: TT.

\section{Importancia del contexto.}

- Afirmación: "El trabajar con problemas del contexto se asume solo como un factor motivador".

Docentes de acuerdo: P14, P17, P18, P21, P22 y P23 — 46\%—.

Tendencia: TT.

- Afirmación: "El aprendizaje real se da en la escuela, aunque se reconozca que fuera de ella se dan pequeños aprendizajes"

Docentes de acuerdo: todos.

Tendencia: TT.

- Afirmación: "Es importante relacionar la enseñanza de un concepto con los contenidos de otras asignaturas"

Docentes de acuerdo: P4, P5, P13, P14, P16, P19 y P20 — 54\%—. 
Tendencia: NT.

\section{Dificultades en el aprendizaje.}

- Afirmación: "Las dificultades en el aprendizaje de una temática se deben en gran parte a problemas cognitivos, de atención o que no se les facilita aprender matemáticas a los estudiantes"

Docentes de acuerdo: P10, P14, P17, P18, P21, P22 y P23 — 54\%—.

Tendencia: TT.

- Afirmación: "Las dificultades de los estudiantes suponen fallas en los métodos de enseñanza del profesor".

Docentes de acuerdo: todos.

Tendencia: TT.

Importancia de los conocimientos previos.

- Afirmación: "Los conocimientos previos de un estudiante sobre una temática son un obstáculo para el aprendizaje”.

Docentes de acuerdo: P10, P14, P17, P18, P22 y P23 — 46\%—.

Tendencia: TT.

- Afirmación: “Es importante que el estudiante proponga conjeturas e hipótesis sobre el resultado de un ejercicio o problema”.

Docentes de acuerdo: P5, P13, P16, P18, P19 y P20 — 46\%—.

Tendencia: NT.

Interrelación entre estudiantes.

- Afirmación: "Los estudiantes solucionan mejor un problema sobre una temática si se les permite intercambiar ideas sobre su resolución".

Docentes de acuerdo: P5, P10, P13, P18, P19 y P20 — 46\%—.

Tendencia: NT.

- Afirmación contraria: "Es preferible que el estudiante trabaje solo, ya que trabajar en grupo es una pérdida de tiempo y no se obtiene rendimiento".

Docentes de acuerdo: P14, P16, P17, P18, P21, P22 y P23 — 54\%—.

Tendencia: TT.

\section{Representación usada por el estudiante.}

- Afirmación: “Es importante que el estudiante use diferentes tipos de representación de los problemas o situaciones, de tal manera quele ayude a comprender la problemática”.

Docentes de acuerdo: P4, P5, P13, P16, P19 y P20 — 46\%—.

Tendencia: NT.

- Afirmación: "Se prioriza el uso de la representación simbólica o matemática y se 
evita otro tipo de representaciones concretas o gráficas”.

Docentes de acuerdo: P10, P14, P17, P18, P21, P22 y P23. — 54\%—.

Tendencia: TT.

\section{Medios educativos.}

- Afirmación: "Los medios educativos facilitan el aprendizaje de los estudiantes".

Docentes de acuerdo: todos.

Tendencia: NT.

\section{Estudio de una operación y su inversa simultáneamente.}

- Afirmación: "Es importante que se enseñe una operación simultáneamente con su inversa ya que permite diferenciar claramente las dos operaciones".

Docentes de acuerdo: P5 y P20 - 15\%—.

Tendencia: NT.

- Afirmación: "Es importante que el estudiante domine primero una operación y luego se estudie su inversa”.

Docentes de acuerdo: el resto.

Tendencia: TT.

\section{Ejercicio o problema.}

- Afirmación: "Para la enseñanza de una temática es preferible partir de una situación problemática”.

Docentes de acuerdo: P5, P10, P13, P16, P18, P19 y P20 — 54\%—.

Tendencia: NT.

- Afirmación: "Hay necesidad de ejercitar la operación y posteriormente colocar los ejercicios de aplicación"

Docentes de acuerdo: el resto.

Tendencia: TT.

\section{Comprensión o mecanización.}

- Afirmación: "Es importante que el estudiante aprenda un método para solucionar una operación, aunque por el momento no lo comprendan”.

Docentes de acuerdo: P10, P17, P18, P21 y P2 - 38\%—.

Tendencia: TT.

- Afirmación contraria: "El estudiante debe comprender lo que está haciendo".

Docentes de acuerdo: el resto.

Tendencia: NT. 


\section{Personal-institucional.}

- Afirmación: "Al estudiante hay que enseñarle un solo método para solucionar un ejercicio o problema. Ese método se debe exigir al estudiante en las evaluaciones".

Docentes de acuerdo: P10, P14, P18, P21, P22 y P23 — 46\%-.

Tendencia: TT.

- Afirmación: "El estudiante debe tener libertad para utilizar el método que considere conveniente a la hora de solucionar problemas o ejercicios. Es más, los docentes deben estimularlos para que apliquen sus propios procedimientos".

Docentes de acuerdo: el resto.

Tendencia: NT.

Se encontró que de las 38 preguntas planteadas, 23 de las respuestas corresponden a una tendencia TT y 15 a NT.

En la siguiente figura se presenta la posición de cada docente frente al número de preguntas con tendencia TT.

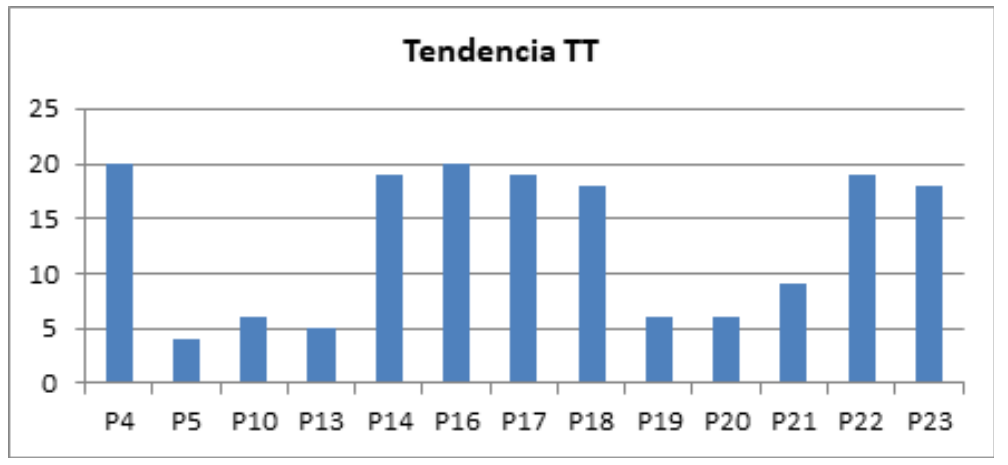

Figura 1. Relación de los docentes con la tendencia TT.

Del anterior gráfico se puede concluir que los docentes P4, P14, P16, P17, P18, P22 y P23 tienen una tendencia TT; es decir, el 54\% de los docentes del estudio se aproximan a una tendencia TT. Aunque — se vuelve a resaltar — ningún docente presenta una única tendencia.

Otro aspecto que se puede destacar es la relación entre las categorías y la tendencia TT, la cual se muestra en la figura 2 .

En las categorías objetivos, significado de la temática, importancia del contexto, dificultades en el aprendizaje, medios educativos, formas de enseñanza de una operación y su inversa, criterio personal o institucional para solucionar problemas, se ve mayoritariamente una tendencia TT; o sea, en el 58\% de las categorías la tendencia predominante es la TT. En el resto de las categorías se ha avanzado hacia metodologías más modernas.

Se resalta que todos los docentes están de acuerdo en las siguientes afirmaciones: para que el estudiante comprenda el significado de un concepto, se deben proponer varios problemas que se solucionen con él; los medios educativos facilitan el aprendizaje; gran parte de las dificultades que tienen los estudiantes en el aprendizaje de cualquier temática se debe a fallas en la enseñanza; el aprendizaje real se da en la escuela, los alumnos aprenden muy 
pocos aspectos fuera de la escuela; el solucionar problemas de la vida diaria es el propósito fundamental de la enseñanza de cualquier concepto. La gran mayoría de las afirmaciones corresponden a una tendencia TT.

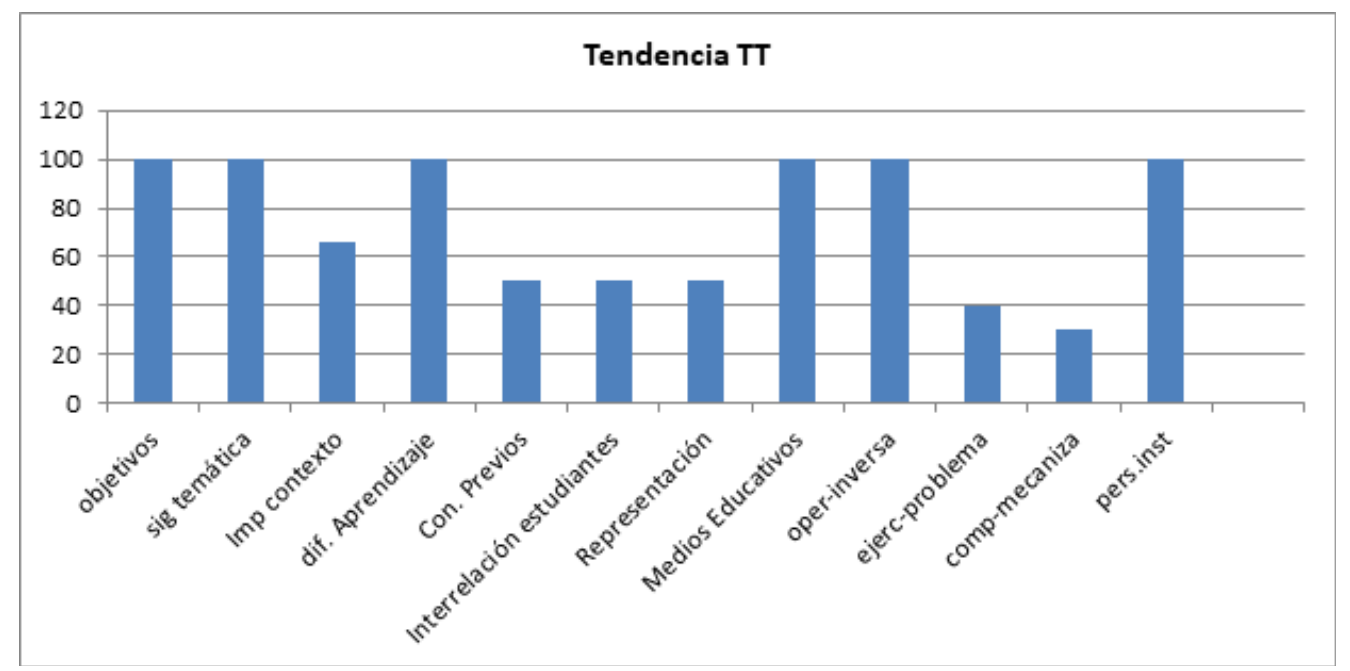

Figura 2. Relación de las categorías frente a la tendencia TT.

Análisis general de las categorías.

Para este proceso se tuvieron en cuenta los docentes que respondieron los dos cuestionarios (tabla 3).

Tabla 3

Resultados de las dos encuestas

\begin{tabular}{ccc}
\hline Código del docente & Cuestionario uno & Cuestionario dos \\
\hline 4 & $\mathrm{C}$ & NT \\
5 & $\mathrm{PC}$ & NT \\
10 & $\mathrm{~N}$ & $\mathrm{TT}$ \\
13 & $\mathrm{E}$ & $\mathrm{NT}$ \\
14 & $\mathrm{~N}$ & $\mathrm{TT}$ \\
16 & $\mathrm{E}$ & $\mathrm{NT}$ \\
17 & $\mathrm{~T}$ & $\mathrm{TT}$ \\
18 & $\mathrm{~N}$ & $\mathrm{TT}$ \\
19 & $\mathrm{E}$ & $\mathrm{NT}$ \\
20 & $\mathrm{C}$ & $\mathrm{NT}$ \\
21 & $\mathrm{~T}$ & $\mathrm{TT}$ \\
22 & $\mathrm{~T}$ & $\mathrm{TT}$ \\
23 & $\mathrm{~N}$ & $\mathrm{TT}$ \\
\hline
\end{tabular}

Nota: C: constructivismo; PC: pedagogía conceptual; N: no define; E: espontaneísmo; T: tecnológica, NT: no tradicional-tecnológica; TT: tradicional-tecnológica.

Se observa que los docentes P17, P21 y P22, que se identificaron inicialmente con tendencia tecnológica, ratifican en la segunda encuesta esa misma tendencia didáctica. Además, los docentes P10, P14, P18 y P23, cuya tendencia didáctica no se pudo identificar en la primera encuesta, se ubicaron en la segunda con tendencia TT; es decir, en definitiva 
el 54\% de los docentes del estudio presentan una tendencia TT. Se aclara que se redujo a 13 el número de docentes, dado que fueron los únicos que decidieron participar de manera voluntaria en todo el proceso del estudio.

\section{Conclusiones}

La mayoría de los docentes del estudio - 54\% - programa sus clases con base en objetivos operacionales, pues buscan una planeación bien completa; evalúan mediante la aplicación de pruebas objetivas, planteadas especialmente a manera de guías; y consideran que el aprendizaje ocurre mediante un proceso progresivo de asimilación de conceptos. De acuerdo con lo anterior, las características de estos profesores se asocian con una tendencia didáctica TT.

En los dos cuestionarios, los docentes tienden en mayoría - el 28\% en el primero y el $54 \%$ en el segundo- hacia lo TT.

En todos los casos se observó que ningún docente aplica una sola tendencia didáctica, sino que poseen rasgos de una amalgama de varias tendencias, pero sí hay una que prima en la mayoría, la TT.

En algunas categorías, se ve la orientación de los docentes hacia la tendencia TT, como en las siguientes: objetivos, significado de la temática, importancia del contexto, dificultades en el aprendizaje, medios educativos, formas de enseñanza de una operación y su inversa, criterio personal o institucional para solucionar problemas.

El uso de los medios educativos es acorde con la tendencia didáctica. Por ejemplo, los profesores que se ubican en tendencias tradicionales utilizan básicamente el tablero o proyectores. Otros docentes, no tradicionales, utilizan la plataforma Moodle de la universidad para diferentes actividades con sus estudiantes, al igual que paquetes informáticos dentro de sus clases, como Derive, Cabri, GeoGebra y otros. Tienen claro que estos son mediadores didácticos.

De acuerdo con los resultados obtenidos en esta parte de la investigación, se espera que nuevas investigaciones muestren de qué manera, al mejorar los procesos comunicativos en el aula y el uso asertivo de los medios educativos, pueden cambiar las tendencias y mejorar las prácticas pedagógicas de los docentes universitarios de matemáticas.

\section{Referencias}

Bruner, J. (1990). Actos de significado. Más allá de la revolución cognitiva (Trad. J. Gómez \& J. Linaza). Madrid: Alianza Editorial.

Chaparro, A., \& Leguizamón, J. (2015). Interacciones sociales en el patio de recreo que tienen el potencial de apoyar el aprendizaje del concepto de probabilidad. Revista Latinoamericana De Etnomatemática, 8(3), 8-24. Recuperado de http://www.revista. etnomatematica.org/index.php/RLE

Chaparro, A., \& Pachón, L. (2016). El razonamiento como eje transversal en la construcción del pensamiento lógico. Praxis \& Saber, 7(14), 219-243. https://doi. org/10.19053/22160159.5224 
Díaz, M. (2010). Encuesta sobre tendencias didácticas de los docentes (Trabajo sin publicar).

Ernest, P. (1989). The knowledge, beliefs and attitudes of the mathematics teacher: A model. Journal of Education for Teaching, 15(1), 13-33.

Gimeno, J. (1982). La pedagogía por objetivos: obsesión por la eficiencia. Madrid: Morata.

Hernández, R., Fernández, C., \& Batista, P. (2006). Metodología de la investigación (4a ed.). México: McGraw Hill.

Jiménez, A. (2019a). La formación de profesores de matemáticas: el caso de la licenciatura más antigua del país. Praxis \& Saber, 10(22), 45-70. https://doi.org/10.19053/22160159. v10.n22.2019.7945

Jiménez, A. (2019b). La dinámica de la clase de matemáticas mediada por la comunicación. Revista de Investigación, Desarrollo e Innovación, 10(1), 121-134. https://10.19053/20278306.v10.n1.2019.10016.

Jiménez, A., Limas, L., \& Alarcón, J. (2016). Prácticas pedagógicas matemáticas de profesores de una institución educativa de enseñanza básica y media. Praxis \& Saber, 7(13), 127152. https://doi.org/10.19053/22160159.4169.

Leguizamón, J. (2017a). Evolución de los patrones de interacción comunicativa de los docentes de matemáticas. Caso UPTC (Tesis doctoral. Universidad Pedagógica y Tecnológica de Colombia, Tunja, Colombia).

Leguizamón, J. (2017b). Patrones de interacción comunicativa del profesor universitario de matemáticas. Un estudio de caso. Praxis \& Saber, 8(16), 57-82. http://dx.doi. org/10.19053/22160159.v7.n15.2016.5723

Leguizamón, J., Patiño, O., \& Suárez, P. (2015). Tendencias didácticas de los docentes de matemáticas y sus concepciones sobre el papel de los medios educativos en el aula. Revista Educación Matemática, 27(3), 154-155.

Marques, R. (2013). A Pedagogia de Jerome Bruner. Recuperado de http://www.eses.pt/usr/ ramiro/ética_e_pedagogia/A_Pedagogia_de_JeromeBruner.pdf

Martínez, M. (2003). Concepciones sobre la enseñanza de la resta: Un estudio en el ámbito de la formación permanente del profesorado (Tesis doctoral, Universidad Autónoma de Barcelona. Barcelona, España).

Ñaupas, H., Mejía, E., Novoa, E., \& Villagómez, A. (2014). Metodología de la investigación cuantitativa-cualitativa y redacción de la tesis. Bogotá: Ediciones desde la U.

Piaget, J. (1978). Problemas de psicología genética. Barcelona: Ariel.

Porlán, R. (1995). Constructivismo y Escuela. Hacia un modelo de enseñanza-aprendizaje basado en la investigación ( $2^{\text {a }}$ ed.). Sevilla: Diada Editorial.

Sierpinska, A. (1998). Three epistemologies, three views of classroom communication: Constructivism, sociocultural approaches, interactionism. En H. Steinbring, M. Bussi, \& A. Sierpinska (Eds.), Language and communication in the mathematics classroom (pp. 30-62). Reston, VA: NCTM.

Strauss, A., \& Corbin, J. (1990). Basics of qualitative research. Grounded theory. Procedures 
and techniques. London: Sage.

Tashakkori, A., \& Teddlie, C (2003). Handbook of Mixed Methods in Social \& Behavioral Research. California: Sage Publications.

Vygotsky, L. (1995). Pensamiento y lenguaje. Teoría del desarrollo cultural de las funciones psíquicas (Trad.M. Rotger). Buenos Aires: Ediciones Fausto.

Zubiría, J. (2006). Los modelos pedagógicos: hacia una pedagogía dialogante (2a ed.). Bogotá: Cooperativa Editorial Magisterio.

Zubiría, M. (2007). Introducción a la Pedagogía Conceptual. Congreso Latinoamericano de Estudiantes de Psicología, Colaepsi. 